\title{
Transcriptomic Analysis on the Regulation of Tomato Ripening by the Ethylene Inhibitor 1-methylcyclopropene
}

\author{
Jamshed Bobokalonov ${ }^{1,2 \dagger}$, Yanhong Liu ${ }^{3 \dagger}$, Tasnuva Shahrin ${ }^{1} \&$ LinShu Liu ${ }^{1 *}$ \\ ${ }^{1}$ Dairy and Functional Foods Research Unit, Eastern Regional Research Center, Agricultural Research Service, \\ United States Department of Agriculture, 600 E. Mermaid Lane, Wyndmoor, PA 19038, USA \\ ${ }^{2}$ Chemistry Institute of the of Sciences of Tajikistan Academy of Sciences, 299/2 Ainy Street, Dushanbe, 734063 \\ Republic of Tajikistan \\ ${ }^{3}$ Molecular Characterization of Foodborne Pathogens Research Unit, Eastern Regional Research Center, \\ Agricultural Research Service, United States Department of Agriculture, 600 E. Mermaid Lane, Wyndmoor, PA \\ 19038, USA \\ $\dagger$ These authors contribute equally to this paper \\ *Correspondence: LinShu Liu, Dairy and Functional Food Research Unit, Eastern Regional Research Center, \\ Agricultural Research Service, United States Department of Agriculture, 600 E. Mermaid Lane, Wyndmoor, PA \\ 19038, USA. Tel: 1-215-233-6486. E-mail: LinShu.Liu@ ars.usda.gov
}

Received: March 20, 2018

Accepted: April 26, $2018 \quad$ Online Published: July 11, 2018

doi:10.5539/jps.v7n2p49

URL: https://doi.org/10.5539/jps.v7n2p49

\begin{abstract}
Tomato is a climacteric fruit whose ripening is regulated by the plant hormone ethylene. 1-methylcyclopropene (1-MCP) is a competitive ethylene inhibitor that can delay the fruit ripening process. To understand the molecular mechanism of how 1-MCP inhibits tomato fruit ripening, transcriptomics (RNA-Seq) was used to identify genes that were differentially expressed in 1-MCP-treated (Day 1) tomato fruits. Of the 35340 genes in the tomato genome, about 50\% were expressed with 1-MCP treatment. There were 5683 genes identified as significantly differentially expressed. Quantitative reverse transcription PCR (qRT-PCR) assays were used to validate the RNA-Seq data. Our results showed that 1-MCP treatment resulted in the down-regulation of fruit ripening-related genes, including genes involved in ethylene synthesis, signal transduction and carotenoid biosynthesis. Our results provide insight at the whole genome level regarding gene regulation by 1-MCP during fruit ripening. Understanding the molecular basis of 1-MCP inhibition on tomato ripening may help farmers and food processors to better use 1-MCP in agriculture and food industry.
\end{abstract}

Keywords: 1-methylcyclopropene (1-MCP), tomato ripening, ethylene inhibitor, RNA-Seq analysis

\section{Introduction}

Fruit ripening is a complex developmental process that coincides with seed maturation. Fruit ripening includes changes in color (loss of green color and increase in non-photosynthetic pigments), texture (cell wall softening), taste (increase in sugar and decrease in organic acids), and flavor (production of volatile compounds). Ripened fruits with aromatic compounds and vivid pigmentation attract animal bites to facilitate their seed dispersal. Fruit ripening is regulated by temperature, gas content in the atmosphere, humidity, and plant hormones such as ethylene (Zhang et al., 2017).

Based on their respiration and ethylene biosynthetic rates, fruits are generally classified into two physiological classes: climacteric and non-climacteric (Osorio et al., 2013). Ethylene plays an important role in climacteric fruit ripening, including in tomatoes, and its function has been well-documented (Bapat et al., 2010). Ethylene is produced in climacteric fruit at the start of ripening. Elevated levels of ethylene bind to the ethylene receptors and activate signal transduction cascades, which lead to activation of a number of genes related to fruit ripening (Giovannoni et al., 2017; Alexander \& Grierson, 2002). Application of exogenous ethylene to unripe tomatoes stimulated fruit ripening whereas inhibitors blocking ethylene synthesis and/or action delayed tomato ripening (Giovannoni, 2001).

It has been known that 1-MCP is an inhibitor of ethylene though blocking of ethylene receptors (Serek et al., 1995). 1-MCP can retard fruit ripening, and therefore, extend the shelf life of fruit (Zhang et al., 2017). Since 
1-MCP is very effective and non-toxic, it has been used widely in the food industry to prolong the post-harvest shelf life of climacteric fruits (Zhang et al., 2017). EthyBloc ${ }^{\circledR}$ and SmartFresh ${ }^{\circledR}$ are the two commercially available products for 1-MCP for ornamentals and fruit and vegetables, respectively. However, the molecular mechanism of 1-MCP inhibition of fruit ripening remains unknown.

Tomato (Solanum lycopersicum) has been used as a good model system to study the mechanism of fruit ripening because it is an important fruit crop for humans, and the tomato genome has been fully sequenced (Sato et al., 2012; Giovannoni, 2001). In addition, tomato has a relatively small genome and well characterized mutants in fruit ripening. Moreover, the ease of transformation in tomato makes mechanistic hypotheses testing feasible using stable transgenic lines (Karlova et al., 2014; Giovannoni et al., 2017).

The advancement of next-generation sequencing (NGS) technologies has revolutionized functional genomic studies. One important application of NGS is to study the transcriptome; the resulting technique is named RNA sequencing (RNA-Seq). This technology has grown rapidly and is replacing microarrays for gene expression studies. RNA-Seq technique involves library construction, sequencing on a NGS platform, and statistical and computational analyses (Wang et al., 2009). RNA-Seq has been applied to differential gene expression (Bloom et al., 2009). RNA-Seq also allows quantification of the relative alterations of each transcript during defined developmental stages or under specific treatment conditions in plants. For example, RNA-Seq was used to study the regulation of auxin and gibberellin (GA), and the effect of abscisic acid (ABA) on fruit ripening (Mou et al., 2016; Tang et al., 2015; Li et al., 2016; Ye et al., 2015). In this study, RNA-Seq was used to study the transcriptome changes under 1-MCP treatment to understand the effect of 1-MCP on tomato fruit ripening at the genomic level. Our research provides insight on ethylene regulation in tomato fruit ripening.

\section{Methods}

\subsection{Tomato Fruits and 1-MCP Treatments}

Mature green tomatoes were purchased from East Coast Fresh Cuts (Savage, MD, USA), received just prior to performing the experiment and used as it is. Tomatoes were treated with SmartFresh ${ }^{\mathrm{TM}}$ (Agrofresh, Spring House, PA, USA) as described previously (Tassoni, et al., 2006), using airtight glass jars to create a "closed system" to generate 1-MCP at a fixed concentration. Each jar was equipped with a Petri dish in the bottom containing a stir bar and $1 \mathrm{~g}$ of the reagent, a tube with one side reaching the dish and another side connected with a $30 \mathrm{ml}$ syringe was fixed with the lid through an airtight rubber horse. All jars were placed on stirrer plates at $22 \pm 1{ }^{\circ} \mathrm{C}$. After loading tomatoes, 5 or 6 (200-300 g per a tomato) in each, the jars were closed, $10 \mathrm{ml}$ of water were injected to each Petri Dish, the stirrer was turned on simultaneously and continued for $24 \mathrm{hr}$. A control experiment was conducted simultaneously without SmartFresh ${ }^{\mathrm{TM}}$. After thus treatment, all tomatoes were removed from the jars, and placed in open space at $22 \pm 1{ }^{\circ} \mathrm{C}$. Pictures were taken at days $0,1,2,3,7,10,13,14$ and 20 .

\subsection{RNA Isolation, Library Preparation, and Illumina Sequencing}

Tomatoes from three groups: 1-MCP-treated (Day1) and untreated (Day 0, and Day 1) were sampled. Pericarps from six tomatoes (two biological replicates from each treatment) were prepared for transcriptomic analysis. Total RNA was extracted from tomato pericarps using the RNeasy Plant Mini Kit (Qiagen, Valacia, CA, USA, according to the manufacturer's instructions. RNA purity was evaluated using a Nanodrop Spectrophotometer (ThermoFisher, Foster City, CA, USA). RNA concentrations were measured using the Qubit ${ }^{\circledR}$ RNA Assay Kit in a Qubit ${ }^{\circledR}$ 2.0 Fluorimeter (Life Technologies, CA, USA), and RNA integrity was assessed using the RNA Nano 6000 Assay Kit of the Agilent Bioanalyzer 2100 system (Agilent Technologies, CA, USA) with RIN numbers between 8.8 and 9.3. Library construction and genome sequencing were performed by Novogene Bioinformatics Technology Co., Ltd. (Beijing, China). A total of $3 \mu \mathrm{g}$ of RNA per sample was used as the initial input material for sequencing library preparation. Sequencing libraries were constructed using the NEBNext Ultra RNA library Prep Kit for Illumina following the manufacturer's instructions. Library concentrations were quantified using a Qubit $^{\circledR} 2.0$ fluorimeter. The insert sizes were evaluated using an Agilent 2100 Bioanalyzer. The libraries were sequenced using an Illumina HiSeq 2000 platform, and 150 bp paired-end reads were obtained.

\subsection{Transcriptome Analysis}

Clean reads generated by filtering low quality reads from the raw data were mapped to a reference tomato ( $S$. lycopersium) genome (http://plants.ensembl.org/biomart/martview;

ftp://ftp.ensemblgenomes.org/pub/release-23/plants/fasta/solanum_lycopersicum/dna/

$\mathrm{ftp} / / / \mathrm{ftp}$.ensemblgenomes.org/pub/release-23/plants/gtf/solanum_lycopersicum/) using TopHat v2.0.12 software. Fragments per kilobase million (FPKM) mapped reads were used to estimate the expression levels (Trapnell, 
2010). Differential expression analysis of two groups was performed using the DESeq R package (Anders et al., 2010). P-values were adjusted by Q-values using the Benjamini-Hochberg method (Benjamini and Hochberg, 1995) to control the false discovery rate. Genes with Q-values < 0.05 were defined as differentially expressed. KOBAS software was used to determine the statistical enrichment of DEGs in the GO terms and KEGG pathways as described previously (Mao et al., 2005). All of the raw sequence data were deposited at the NCBI BioProject database with Bio Project ID number PRJNA413422.

\subsection{Real-time Quantitative Reverse Transcription Polymerase Chain Reaction (qRT-PCR) Assays}

Synthesis of cDNA was carried out as described previously (Liu et al., 2011). Primers ordered from Integrated DNA Technologies (IDT) (www.idtdna.com) are listed in Table 1.

qRT-PCR was performed in a 96-well plate on a Quant Studio 6 Flex real-time PCR system (ThermoFisher, Foster City, CA). PCR reactions were carried out in a $50 \mu 1$ total volume containing $25 \mu 1$ Power SYBR Green

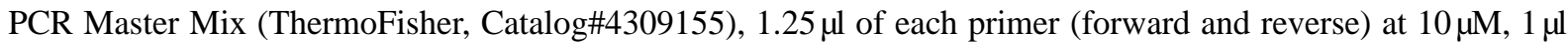
of cDNA and nuclease-free water (ThermoFisher, Catalog\#AM9938). Thermal cycling parameters were as described previously (Liu et al., 2011). Results were visualized using the Quant Studio Real-Time PCR Software v1.3 provided with the Quant Studio 6 Flex Real-Time PCR System. To determine relative gene expression, the $C t$ value of the internal control gene (actin) was subtracted from the treated and untreated samples. The $\Delta \mathrm{Ct}$, $\Delta \Delta \mathrm{Ct}$, and the $2^{-\mathrm{fx}}$ values were calculated as described previously (Pfaffl 2001).

Table 1. Primer sequences used in qRT-PCR assays

\begin{tabular}{lll}
\hline Genes & Forward primers & Reverse primers \\
\hline Solyc04g051360.2.1 & TGATTTCTAGGGGTGTTGGA & TTGAACCTTCACCACCTGAT \\
Solyc05g054890.2.1 & TACGAAATGCTGCAAAAACA & CAAGACCTGAAGTTGGTTCG \\
Solyc11g072600.1.1 & TTCCGATGGATAACAATGCT & GCACCTCTCGGAAACTAACA \\
Solyc07g053410.2.1 & GAACCCCTTCAGTCCAATTT & GCTGGCACTTGAAAATCAGT \\
Solyc05g050830.1.1 & CGGCTCACCTTAATTTCCT & TTCCTCTTCCTCGTCTTCCT \\
Solyc03g095980.2.1 & CAAAACAGCAGAAATCAGCA & ATCATCGCAACAAAAAGAGG \\
Solyc04g014400.2.1 & CCTGAGCAAAATACGAGCAT & CTGTTGTGGGATAGGTCCAG \\
Solyc03g025350.2.1 & GCTGGTGGTGTAGCAGTTCT & ACTTGCTTCGGATATTGCAC \\
Solyc09g089610.2.1 & GTGATTGTCGGATTCCAAAG & AACCTCCACACCACGTAAAA \\
Solyc08g078180.1.1 & TTGAGGATTAATTCCGGTGA & GAGCAACCTTCTTCCTCCTC \\
Solyc07g049550.2.1 & ATGGGACTCGGATGTCACTA & TTTCGGATAAACTTGCTTGC \\
Solyc11g012980.1.1 & AATGAGAAGCTGGGGATCAT & AAAAGTGCAGCATCATAGGC \\
Solyc11g010710.1.1 & GCCATAAAGTTCAGGGGAGT & GCTCGATGATGATTTCTGCT \\
Solyc08g078170.1.1 & TCGGATCCTGTTATTCCAAA & AACAGATTGTGCTGGTGGTT \\
Solyc06g053260.1.1 & CCCTTGTCATGTCGAAGAAT & TCAGGCCTTATAGCACCAAG \\
\hline
\end{tabular}

\subsection{Statistical Analysis}

Student's t-test was used to determine the significant differences between samples.

\section{Results}

\subsection{Effects of 1-MCP on Tomato Fruit Ripening}

To test the effect of 1-MCP on tomato fruit ripening, 1-MCP was used to treat the mature green tomato fruits. As shown in Figure 1, a dramatic delay in color alteration was observed in 1-MCP-treated tomato fruits. Control tomatoes started to turn orange at Day 3, and turned completely red at Day 6. The 1-MCP-treated tomato fruits started to turn orange at Day 10, and turned completely red at Day 20 (Figure 1). Changes in fruit color were delayed by 14 days after 1-MCP treatment. 


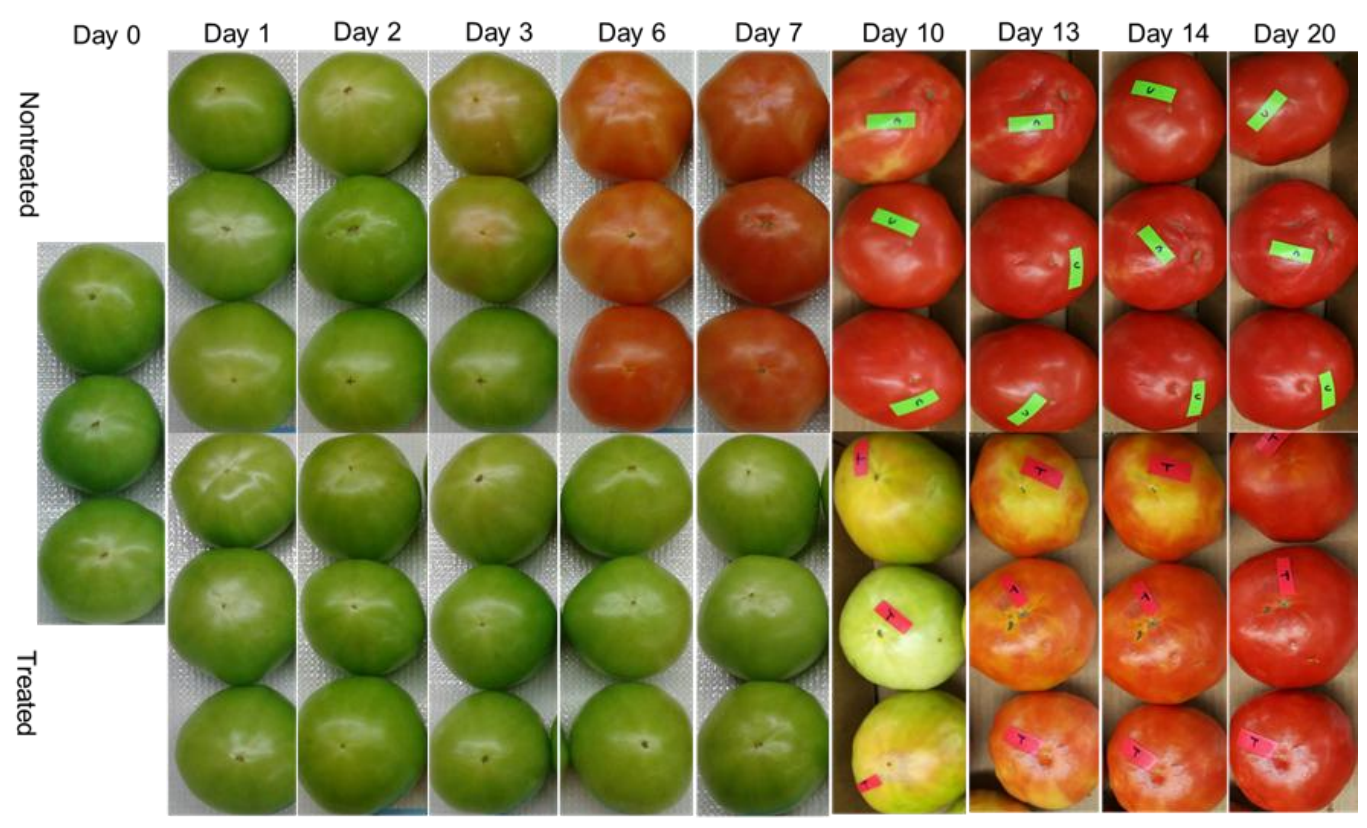

Figure 1. Color changes for 1-MCP treated and non-treated tomatoes. Mature green tomatoes were treated with 1-MCP for 24 hours and compared with untreated tomatoes. Pictures were taken at

Day $1,2,3,6,7,10,13,14$, and 20

\subsection{Summary of Transcriptomics Data}

To investigate the effect of 1-MCP on tomato fruit ripening, pericarps from tomato fruits in both 1-MCP-treated and untreated groups were sampled at Day 1 after treatment. Pericarps from the control group before treatment (Day 0) were also collected. RNA was extracted and subjected to transcriptomic analysis. The raw sequence reads obtained by Illumina sequencing were from 73.7 to 123 million reads per sample, and over $97 \%$ had a quality score higher than 20 . After data filtration, 71.7 to 120 million clean reads were gathered; roughly $50 \%$ of the clean reads were uniquely mapped to the tomato reference genome (Table 2).

Hierarchical cluster analysis was used to represent variances in DEGs (Figure 2A). Among more than 35340 genes with different reads detected, roughly $50 \%$ of them had a fragments per kilobase million (FPKM) $>1$. Of the 5683 differentially expressed genes (DEGs), 3066 genes were up-regulated and 2617 were down-regulated (Figure 2B). Of these DEGs, 2088 were significantly changed (more than 2-fold) compared to the untreated day 1 (Con-D1) samples. There were 1214 genes up-regulated whereas 874 genes were down-regulated in 1-MCP-treated samples (Tre-D1).

Table 2. Throughput and quality of RNA-Seq data

\begin{tabular}{lllllll}
\hline $\begin{array}{l}\text { Sample } \\
\text { name }\end{array}$ & Raw reads & $\begin{array}{l}\text { Clean } \\
\text { reads }\end{array}$ & $\begin{array}{l}\text { Q20 } \\
(\%)^{*}\end{array}$ & Total mapped & Multiple mapped & Uniquely mapped \\
\hline U0-1 & 73720680 & 71720882 & 97.5 & $65851125(91.82 \%)$ & $1028752(1.43 \%)$ & $64822373(90.38 \%)$ \\
$\mathrm{U} 0-2$ & 81324926 & 78662492 & 97.55 & $72002946(91.53 \%)$ & $1097549(1.4 \%)$ & $70905397(90.14 \%)$ \\
$\mathrm{U} 1-1$ & 118771830 & 114590170 & 98.3 & $64748336(56.5 \%)$ & $1024390(0.89 \%)$ & $63723946(55.61 \%)$ \\
$\mathrm{U} 1-3$ & 122902580 & 119541298 & 98.16 & $65657537(54.92 \%)$ & $1092604(0.91 \%)$ & $64564933(54.01 \%)$ \\
T1-1r & 123401776 & 119975950 & 97.95 & $60089836(50.08 \%)$ & $692821(0.58 \%)$ & $59397015(49.51 \%)$ \\
T1-2r & 113749964 & 110636956 & 98.28 & $56339418(50.92 \%)$ & $617191(0.56 \%)$ & $55722227(50.36 \%)$ \\
\hline
\end{tabular}

*Q20 means that the percentages of bases whose correct base recognition rates are greater than $99 \%$ in total base. U0-1 and U0-2 represent untreated tomatoes (Day 0). U1-1 and U1-3 represent untreated tomatoes (Day1) whereas $\mathrm{T} 1-1 \mathrm{r}$ and $\mathrm{T} 1-2 \mathrm{r}$ represent 1-MCP treated tomatoes (Day1). 


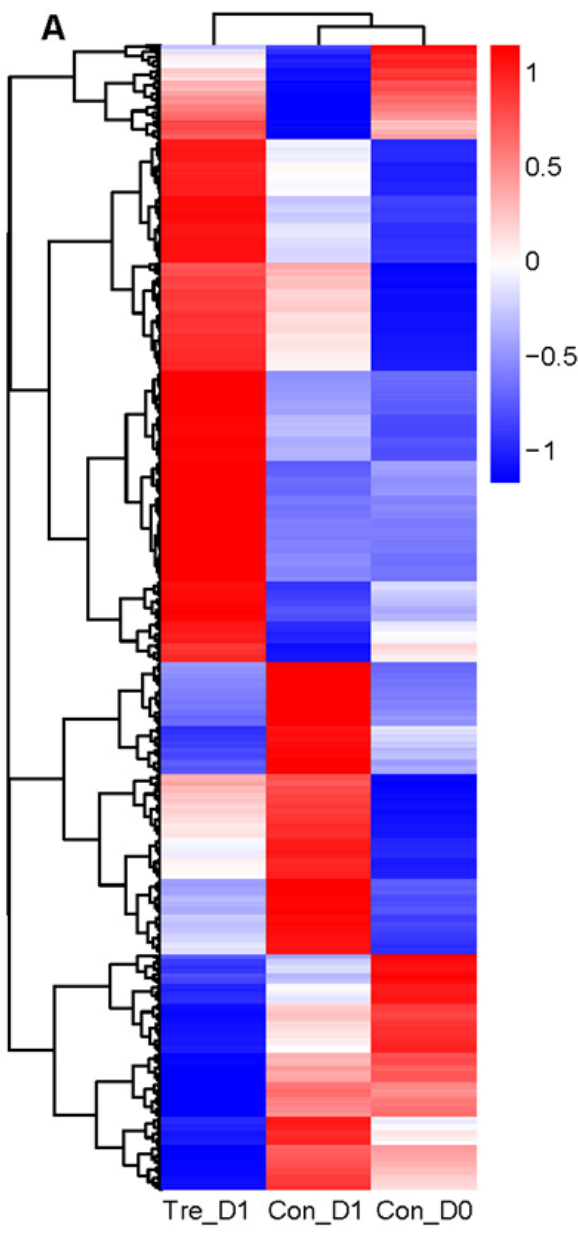

B

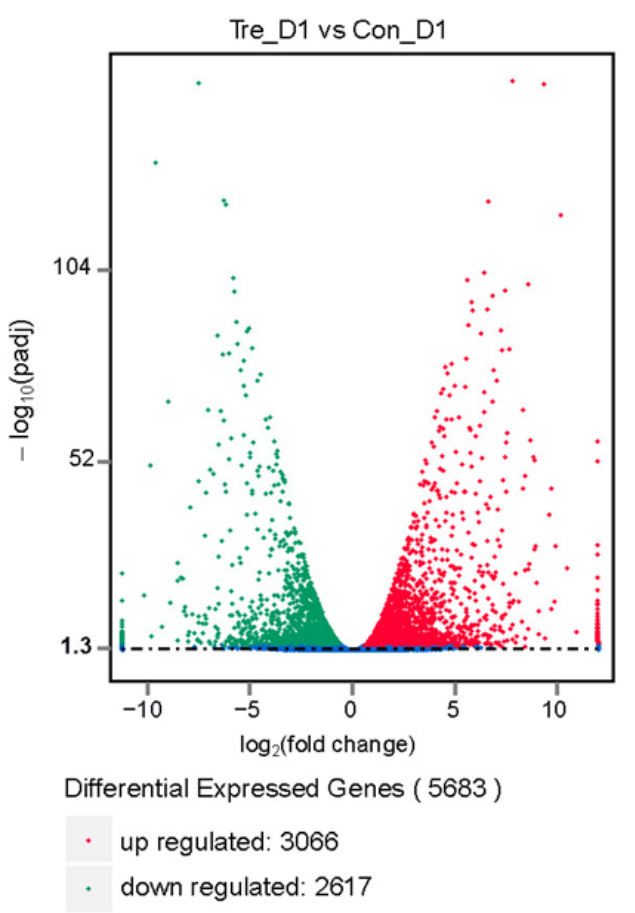

Figure 2. Differentially expressed genes (DEGs) in the MCP-treated samples (Day1). (A) Hierarchical clustering and heat map of differentially expressed genes based on the expression levels (RPKM) in Con-D1, Tre-D1 and Con-D1 samples. Con-D0 represents untreated tomatoes before 1-MCP treatment. Con-D1 represents untreated tomatoes (Day1), whereas Tre-D1 represents 1-MCP treated tomatoes (Day 1). Red and blue colors represent high and low expressed genes, respectively. (B) Volcano plot showing the numbers of significantly differentially expressed genes in 1-MCP treated (Day1) and untreated (Day1) tomatoes. Red and Green colors represent up-

and down-regulated genes, respectively. Data presented here are averages of two biological replicates

\subsection{Gene Ontology (GO) Enrichment and Kyoto Encyclopedia of Genes and Genomes (KEGG) Pathway Enrichment Analysis of DEGs}

GO enrichment analysis was used to study the distribution of DEGs for gene function in terms of biological process (BP), cellular component (GC) and molecular function (MF). There were $148 \mathrm{GO}$ categories that were differentially enriched (corrected p-value <0.05). Of the thirty GO terms (BP: 16 terms, CC: 12 terms, MF: 3 terms) significantly enriched in 1-MCP-treated (Day1) compared with untreated (Day1) shown in Figure 3A, the most enriched terms were DNA packaging complex (GO: 0044815, 44 DEGs), photosynthesis, and light harvesting (GO: 0009765, 30 DEGs) in CC and BP. All DEGs were also aligned to KEGG pathways to identify major pathways (Figure 3B). In the 1-MCP-treated (TreD1) samples, DEGs were matched in $120 \mathrm{KEGG}$ metabolic pathways. The top four enriched pathways including the highest numbers of DGEs were: Metabolic pathways (586 DEGs), Biosynthesis of secondary metabolites (320 DGEs), Carbon metabolism (90 DGEs) and Plant hormone signal transduction (86 DGEs). There were thirteen pathways that had p-values $<0.05$. 

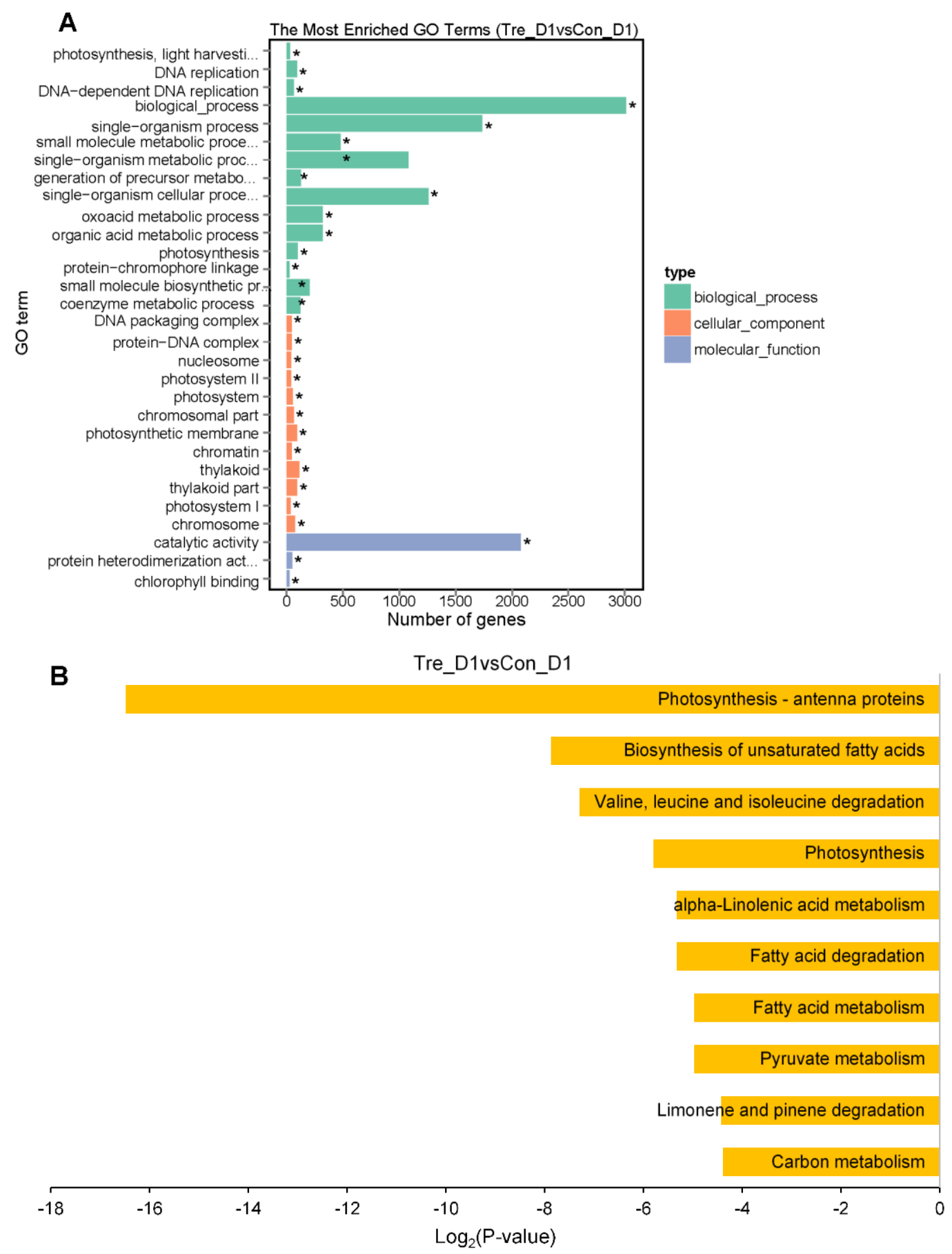

Figure 3. GO and KEGG enrichment analysis of DEGs in 1-MCP-treated tomatoes. (A) The top thirty most enriched GO terms in 1-MCP treated (Tre-D1) compared to Con-D1. The x-axis is the number of differentially expressed genes and the y-axis is GO terms enriched. Asterisks (*) indicate significantly enriched GO terms.

Different colors are used to identify biological process. (B) The top ten most enriched KEGG pathways in 1-MCP treated (Tre-D1) compared to untreated (Con-D1) tomatoes

\subsection{Analysis of Genes Involved in Ethylene Biosynthesis and Signal Transduction Pathways}

To investigate whether 1-MCP inhibited ethylene synthesis, genes involved in ethylene pathways were extracted from transcriptomic data (with adjusted $\mathrm{p}$ value <0.05) and compared (Figure 4 and Table 3 ). The expression level of two S-adenosylmethionine synthase genes (Solyc10g083970 and Solyc09g008280) was 3.14 and 7.04-fold elevated, respectively, whereas the expression level of the S-adenosylmethionine synthase 1 gene 
(Solyc01g101060) was not altered significantly. Three genes (Solyc01g095080, Solyc08g081550, and Solyc08g081540) encoding for 1-aminocyclopropane-1-carboxylate synthase were all up-regulated 6 to 8 -fold whereas one gene was down-regulated significantly. Of the 5 genes encoding for 1-aminocyclopropane-1-carboxylate oxidase, the expression level of one gene (Solyc02g081190) encoding for 1-aminocyclopropane-1-carboxylate oxidase 4 was 5.6-fold up-regulated whereas the expression levels of other three genes (Solyc09g089580, Solyc07g049550 and Solyc07g026650) were significantly down-regulated (0.02-0.1 fold).

In our study, the expression levels of two ethylene receptors, ethylene receptor homolog (ETR4) (Solyc06g053710) and Never ripe (Solyc09g075440) were significantly down regulated $(0.07$ and 0.15 , respectively) in 1-MCP-treated tomato fruits. Our data indicate that 1-MCP binds to specific ethylene receptors, possibly ETR4 and Never ripe, therefore reducing their production.

The ethylene signal transduction pathway ultimately leads to the activation of transcriptional regulators belonging to the Ethylene Response Factor (ERF) family of transcription factors. In our study, of the 9 ERFs, 5 of them (Solyc07g008250, Solyc12g009560, Solyc01g065980, Solyc02g077370 and Solyc09g066360) were significantly down-regulated (0.02 to 0.15) whereas two of them (Solyc04g051360 and Solyc04g014530) were significantly up-regulated (350 and 72-fold, respectively) in 1-MCP-treated tomato fruits. Our data suggest that 1-MCP delayed ripening by inhibiting ERFs, therefore inhibiting ripening-related gene expression.

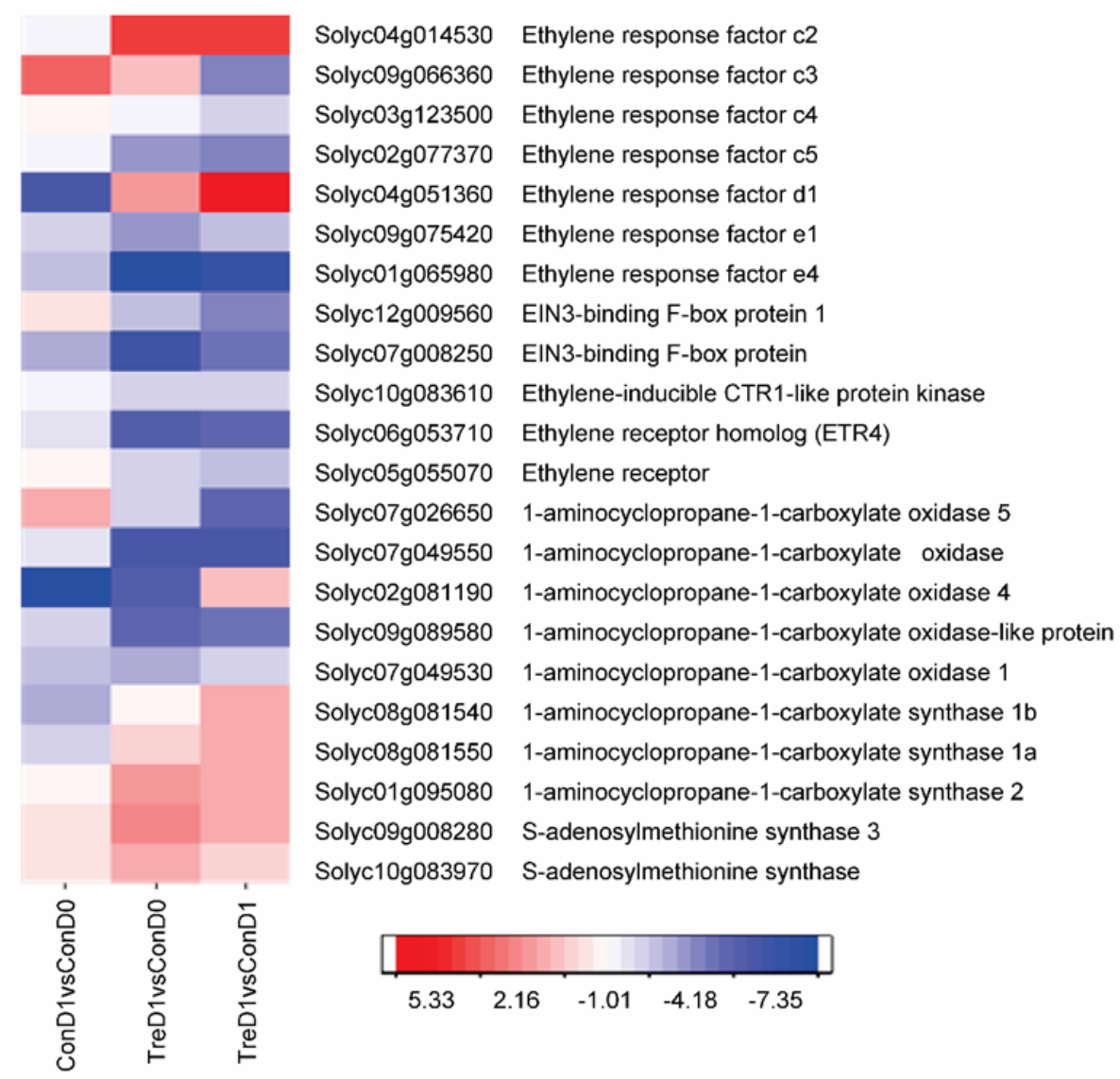

Figure 4. Heat map of the differentially expressed genes (DEGs) in ethylene synthesis and signal transduction after 1-MCP treatment. The RPKM values were normalized and converted to Z-scores to scale the gene expression levels. Red and blue colors indicate high and low expression, respectively 
Table 3. DEGs involved in ethylene synthesis and signal transduction

\begin{tabular}{llll}
\hline Gene ID & Annotation & \multicolumn{2}{l}{ TreD1vsConD1 } \\
\cline { 3 - 4 } & & Fold change & P value \\
\hline Solyc10g083970 & S-adenosylmethionine synthase & 3.14 & 0.001 \\
Solyc09g008280 & S-adenosylmethionine synthase 3 & 7.04 & $1.70 \mathrm{E}-08$ \\
Solyc01g101060 & S-adenosylmethionine synthase 1 & 1.37 & 0.05 \\
Solyc01g095080 & 1-aminocyclopropane-1-carboxylate synthase 2 & 6.99 & $2.43 \mathrm{E}-25$ \\
Solyc08g081550 & 1-aminocyclopropane-1-carboxylate synthase 1a & 8.16 & 0.05 \\
Solyc08g081540 & 1-aminocyclopropane-1-carboxylate synthase 1b & 6.69 & $8.77 \mathrm{E}-10$ \\
Solyc07g049530 & 1-aminocyclopropane-1-carboxylate oxidase 1 & 0.54 & 0.05 \\
Solyc09g089580 & 1-aminocyclopropane-1-carboxylate oxidase-like protein & 0.10 & $4.89 \mathrm{E}-49$ \\
Solyc02g081190 & 1-aminocyclopropane-1-carboxylate oxidase 4 & 5.58 & 0.0001 \\
Solyc07g049550 & 1-aminocyclopropane-1-carboxylate oxidase & 0.03 & $1.62 \mathrm{E}-91$ \\
Solyc07g026650 & 1-aminocyclopropane-1-carboxylate oxidase 5 & 0.07 & $4.30 \mathrm{E}-48$ \\
Solyc05g055070 & Ethylene receptor & 0.38 & $1.48 \mathrm{E}-10$ \\
Solyc06g053710 & Ethylene receptor homolog (ETR4) & 0.07 & $2.83 \mathrm{E}-60$ \\
Solyc09g075440 & Never ripe & 0.15 & $2.38 \mathrm{E}-12$ \\
Solyc10g083610 & Ethylene-inducible CTR1-like protein kinase & 0.55 & $2.22 \mathrm{E}-05$ \\
Solyc07g008250 & EIN3-binding F-box protein & 0.10 & $2.26 \mathrm{E}-46$ \\
Solyc12g009560 & EIN3-binding F-box protein 1 & 0.15 & $2.78 \mathrm{E}-05$ \\
Solyc01g065980 & Ethylene response factor e4 & 0.02 & $1.78 \mathrm{E}-105$ \\
Solyc09g075420 & Ethylene response factor e1 & 0.42 & $3.07 \mathrm{E}-08$ \\
Solyc04g051360 & Ethylene response factor d1 & 350.08 & $1.84 \mathrm{E}-05$ \\
Solyc02g077370 & Ethylene response factor c5 & 0.15 & 0.0003 \\
Solyc03g123500 & Ethylene response factor c4 & 0.59 & 0.0002 \\
Solyc09g066360 & Ethylene response factor c3 & 0.13 & 0.008 \\
Solyc04g014530 & Ethylene response factor c2 & 71.82 & $1.06 \mathrm{E}-07$ \\
\hline
\end{tabular}

\subsection{Analysis of Genes Involved in Carotenoid Biosynthesis in 1-MCP-treated Tomato Fruits}

The expression levels of the genes encoding the enzymes associated with carotenoid metabolism were investigated (Table 4). The expression level of the gene encoding for phytoene synthase 1 ( $p s y 1$ ) (Solyc03g031860) was significantly inhibited (only 8\% compared to untreated) in 1-MCP treated tomato fruits. PSY1 catalyzes the first critical step in the carotenoid biosynthetic pathway, and down-regulation of this gene abolishes normal carotenoid accumulation (Bartley et al., 1992; Fray and Grierson, 1993). On the other hand, the expression level of the gene (Solyc03g007960) encoding for Beta-carotene hydroxylase-2 was 13-fold elevated (Table 4).

Table 4. DEGs associated with carotenoid metabolism

\begin{tabular}{llll}
\hline \multirow{2}{*}{ Gene ID } & Annotation & \multicolumn{2}{c}{ TreD1vsConD1 } \\
\cline { 3 - 4 } & & Fold change & P value \\
\hline Solyc03g031860 & Phytoene synthase 1 & 0.08 & $7.27 \mathrm{E}-57$ \\
Solyc04g040190 & Lycopene beta-cyclase & 1.81 & 0.01 \\
Solyc03g007960 & Beta-carotene hydroxylase-2 & 13.03 & $3.70 \mathrm{E}-41$ \\
\hline
\end{tabular}

\subsection{Analysis of Genes Involved in Cell Wall Degradation}

The expression levels of genes involved in cell wall degradation were investigated in the RNA-Seq experiments (Table 5). Of the 8 genes related with cell wall metabolism, Solyc07g017600 and Solyc09g010210 encoding for pectinesterase and Endo-1, 4-beta-glucanase precursor were highly expressed in 1-MCP-treated tomato fruits. 
Table 5. DEGs associated with cell wall degradation

\begin{tabular}{llll}
\hline Gene ID & Annotation & \multicolumn{2}{l}{ TreD1vsConD1 } \\
\cline { 3 - 4 } & & Fold change & P value \\
\hline Solyc07g017600 & Pectinesterase & 38.468 & $9.03 \mathrm{E}-24$ \\
Solyc09g010210 & Endo-1,4-beta-glucanase precursor & 60.597 & $8.32 \mathrm{E}-05$ \\
Solyc02g091680 & Probable beta-D-xylosidase 6-like & 0.620 & 0.01 \\
Solyc01g104950 & Beta-xylosidase & 0.245 & 0.01 \\
Solyc10g047030 & Beta-D-xylosidase 1 precursor & 0.109 & 0.04 \\
Solyc09g005850 & Probable pectate lyase 4-like & 2.170 & $1.86 \mathrm{E}-05$ \\
Solyc03g031840 & Expansin precursor & 1.635 & 0.02 \\
Solyc06g051800 & Expansin 1 & 2.453 & 0.02 \\
\hline
\end{tabular}

\subsection{Analysis of Genes Involved in Cell Wall Degradation}

DNA methylation has been shown to be involved in fruit ripening (Gallusci et al., 2016). Genomic DNA cytosine methylation from tomato pericarps was decreased prior to ripening initiation (Cao et al., 2014). The expression level of Solyc12g100330.1 was 17-fold elevated in 1-MCP treated tomato fruits (Table 6). Solyc12g100330.1 encodes for cytosine-5 DNA methyltransferase (C5-MTase) in tomato. High expression of this gene may contribute to maintaining of high DNA methylation levels in the nucleus, which delayed fruit ripening.

Table 6. DEGs associated with DNA methylation

\begin{tabular}{llll}
\hline \multirow{2}{*}{ Gene ID } & Annotation & \multicolumn{2}{l}{ TreD1vsConD1 } \\
\cline { 3 - 4 } & & Fold change & P value \\
\hline Solyc12g100330.1 & Cytosine-specific methyltransferase & 1.82 & 0.0002 \\
Solyc01g006100.2 & Cytosine-specific methyltransferase & 17.17 & $3.43 \mathrm{E}-08$ \\
Solyc08g005400.2 & Cytosine-specific methyltransferase & 2.18 & $3.74 \mathrm{E}-05$ \\
Solyc02g062740.2 & DNA (Cytosine-5-)-methyltransferase 3 & 0.67 & 0.008 \\
Solyc05g053260.2 & DNA methyltransferase & 0.68 & 0.03 \\
Solyc09g009080.2 & Repressor of silencing 1 & 2.91 & $4.04 \mathrm{E}-09$ \\
Solyc11g007580.1 & HhH-GPD family protein & 1.54 & 0.006 \\
\hline
\end{tabular}

\subsection{Validation of Selected DEGs by qRT-PCR}

RT-PCR was used to validate the gene expression data obtained from RNA-Seq experiments. Fifteen genes with different expression levels were randomly selected from transcriptomic data and tested by qRT-PCR assays. The expression levels of all tested genes in 1-MCP treatments showed similar expression tendency between RNA-Seq and qRT-PCR, and there was a high correlation $\left(\mathrm{R}^{2}=0.91\right)$ between qRT-PCR and RNA-Seq data (Figure 5). Our data suggest that the transcriptomic data obtained from RNA-Seq experiments were very reliable.

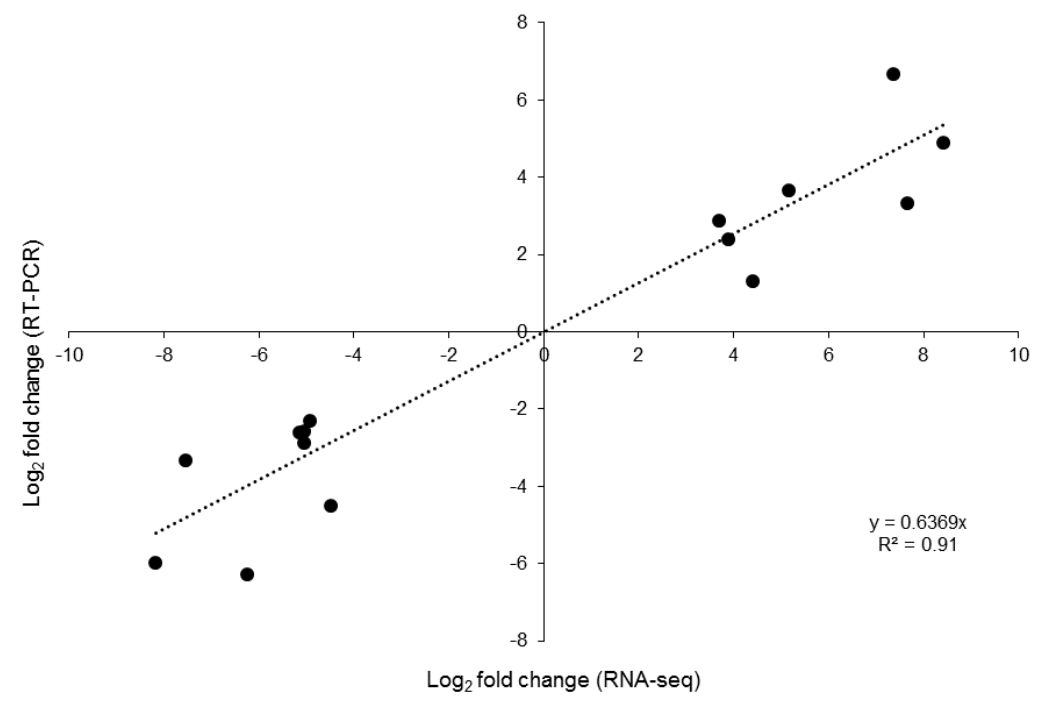

Figure 5. Correlation of gene expression data from RNA-Seq and qRT-PCR 


\section{Discussion}

The ethylene biosynthesis pathway is well studied in higher plants (Giovannoni et al., 2017). S-adenosylmethinin synthase, aminoclyclopr opane-1-carboxylate synthase, and 1-aminocyclopropane-1-carboxylate oxidase are the three key enzymes involved in ethylene synthesis. Although the expression levels of genes encoding for S-adenosylmethionine synthase and 1-aminocyclopropane-1-carboxylate synthase were higher in 1-MCP treated fruits, the expression levels of three genes encoding for 1-aminocyclopropane-1-carboxylate oxidase were inhibited significantly. The down-regulation of the gene encoding for 1-aminocyclopropane-1-carboxylate oxidase may influence the low production of ethylene. Our data were consistent with previous data showing that the production of ethylene was inhibited by 1-MCP in tomato fruits (Tassoni et al., 2006).

Ethylene regulates fruit ripening through binding to ethylene receptors and activating signal transduction pathways (Alexander \& Grierson, 2002). The ethylene signal transduction pathway is negatively regulated by ethylene receptors (Hua \& Meyerowitz, 1998). In the absence of ethylene, ethylene receptors actively inhibit ethylene responses. After ethylene binds to receptors, the receptor undergoes a conformational change from the active to inactive state, suppression is removed, and the ethylene response occurs. There are six known receptors in tomato (Klee \& Tieman, 2002). Previous studies suggested that 1-MCP inhibited ethylene function by competing with ethylene receptors (Sisler, 2006; Serek et al., 1995). A model was proposed for 1-MCP action. In this model, 1-MCP binds irreversibly to ethylene receptors, preventing ethylene binding and keeping the receptor in the active signaling state, therefore, inhibiting ripening (Binder \& Bleecker, 2003). This model was supported by the fact that 1-MCP inhibited gene expression of ethylene receptors.

The color of the pigment in tomato fruits is determined mainly by chlorophyll and carotenoids. In tomato ripening, the color of tomatoes changes from green to red with pigmentation containing carotenoids and lycopene. Tomato ripening is followed by elevated transcription of several genes encoding enzymes involved in the biosynthesis of carotenoids (Bramley, 2002). Although there was no color difference between 1-MCP treated (Day 1) and untreated tomatoes (Day 1) (Figure 1), the reduced expression of Solyc03g031860 correlated with the significant color change delay in 1-MCP-treated tomato fruits in later stages (Figure 1). In addition, 1-MCP has been shown to inhibit carotenoid biosynthesis in papaya (Fabi et al., 2007; Shen et al., 2017). 1-MCP also inhibited the expression of the psy gene and carotenoid accumulation in apricot (Kita et al., 2007). $\beta$-carotene hydroxylase- 2 catalyzes hydroxylation reactions that convert beta-carotene to zeaxanthin. Although majority of carotenoid biosynthesis is regulated at the transcriptional level, post-transcriptional regulation, including feedback inhibition has also been reported (Bramley, 2002). The lack of correlation at the gene expression level may indicate some post-transcriptional regulation.

Fruit ripening is also associated with cell wall softening. Pectinesterase is an important enzyme responsible for pectin demethylation that is involved in cell-wall softening. Silencing of this gene in tomato has been shown to enhance softening in fruit ripening (Phan et al., 2007). Since 1-MCP inhibited fruit ripening, its expression was also elevated. In agreement with our gene expression data, the protein level of pectinesterase was also elevated after 1-MCP treatment in papaya fruit (Huerta-Ocampo et al., 2012).

Our results showed that 1-MCP treatment results in the down-regulation of a number of fruit ripening related genes, such as those involved in ethylene synthesis and signal transduction, cell wall synthesis, and pigment synthesis. This represents the first transcriptomic study of the effect of 1-MCP in tomato fruits. Our results also provide insight at the genome level on gene regulation by 1-MCP during fruit ripening and provide a better understanding of the role of ethylene in tomato ripening. Understanding the molecular basis of MCP action can help farmers and food processors to better use 1-MCP in the food industry.

\section{Acknowledgments}

We would like to thank Dr. Pina Fratamico for critical reading of manuscript, Amy Ream for performing qRT-PCR experiments and Audrey Thomas-Gahring for purchasing tomato fruits and sample shipments.

Mention of trade names or commercial products is solely for the purpose of providing specific information and does not imply recommendation or endorsement by the U.S. Department of Agriculture. USDA is an equal opportunity employer.

\section{References}

Alexander, L., \& Grierson, D. (2002). Ethylene biosynthesis and action in tomato: a model for climacteric fruit ripening. J Exp Bot, 53, 2039-55. https://10.1093/jxb/erf072

Anders, S., \& Huber, W. (2010). Differential expression analysis for sequence count data. Genome Biol. (DESeq). Genome Biol, 11, R106. https://doi.org/10.1186/gb-2010-11-10-r106 
Bapat, V. A., Trivedi, P. K., Ghosh, A., Sane, V. A., Ganapathi, T. R., \& Nath, P. (2010). Ripening of fleshy fruit: molecular insight and the role of ethylene. Biotechnol Adv, 28, 94-107. https://doi.org/10.1016/j.biotechadv.2009.10.002

Bartley, G. E., Viitanen, P. V., Bacot, K. O., \& Scolnik, P. A. (1992). A tomato gene expressed during fruit ripening encodes an enzyme of the carotenoid biosynthesis pathway. J Biol Chem, 267, 5036-5039

Benjamini, Y., \& Hochberg, Y. (1995). Controlling the False Discovery Rate: A Practical and Powerful Approach to Multiple Testing. Journal of the Royal Statistical Society. Series B (Methodological), 57, 289-300.

Binder, B. M., \& Bleecker, A. B. (2003). A model for ethylene receptor function and 1-methylcyclopropene action. Acta Hort, 628, 177-187. https://doi.org/10.17660/ActaHortic.2003.628.21

Bloom, J. S., Khan, Z., Kruglyak, L., Singh, M., \& Caudy, A. A. (2009). Measuring differential gene expression by short read sequencing: quantitative comparison to 2-channel gene expression microarrays. BMC Genomics, 10, 1-10. https://doi.org/10.1186/1471-2164-10-221

Bramley, P. M. (2002). Regulation of carotenoid formation during tomato fruit ripening and development. Fruit Development and Ripening Special Issue. J Exp Bot, 53, 2107-2113. https://doi.org/10.1093/jxb/erf059

Cao, D., Ju, Z., Gao, C., Mei, X., Fu, D., Zhu, H., Luo, Y., \& Zhu, B. (2014). Genome-wide identification of cytosine-5 DNA methyltransferases and demethylases in Solanum lycopersicum. Gene, 550, 230-237. https://doi.org/10.1016/j.gene.2014.08.034

Fabi, J. P., Cordenunsi, B. R., De Mattos Barreto, G. P., Mercadante, A. Z., Lajolo, F. M., \& Oliveira do Nascimento, J. R. (2007). Papaya fruit ripening: response to ethylene and 1-methylcyclopropene (1-MCP). J Agric Food Chem, 55, 6118-23. https://doi.org/10.1021/jf070903c

Fray, R. G., \& Grierson, D. (1993). Identification and genetic analysis of normal and mutant phytoene synthase genes of tomato by sequencing, complementation and co-suppression. Plant Mol Biol, 22, 589-602. https://doi.org/10.1007/BF00047400

Gallusci, P., Hodgman, C., Teyssier, E., \& Seymour, G. B. (2016). DNA methylation and chromatin regulation during fleshy fruit development and ripening. Front Plant Sci., 7, 807. https://doi.org/10.3389/fpls.2016.00807

Giovannoni, J. (2001). Molecular biology of fruit maturation and ripening. Annu Rev Plant Physiol Plant Mol Biol, 52, 725-749. https://doi.org/10.1146/annurev.arplant.52.1.725

Giovannoni, J., Nguyen, C., Ampofo, B., Zhong, S., \& Fei, Z. (2017). The epigenome and transcriptional dynamics of fruit ripening. Annu Rev Plant Biol, 68, 61-84. https://doi.org/10.1146/annurev-arplant-042916-040906

Hua J, Meyerowitz EM. (1998). Ethylene responses are negatively regulated by a receptor gene family in Arabidopsis thaliana. Cell. 24;94(2):261-71.

Huerta-Ocampo, J. Á., Osuna-Castro, J. A., Lino-López, G. J., Barrera-Pacheco, A., Mendoza-Hernández, G., De León-Rodríguez, A., \& Barba de la Rosa, A. P. (2012). Proteomic analysis of differentially accumulated proteins during ripening and in response to 1-MCP in papaya fruit. J Proteomics, 75, 2160-2169.

https://doi.org/10.1016/j.jprot.2012.01.015

Karlova, R., Chapman, N., David, K., Angenent, G. C., Seymour, G. B., \& De Maagd, R. A. (2014). Transcriptional control of fleshy fruit development and ripening. J Exp Bot 65, 4527-41. https://doi.org/10.1093/jxb/eru316

Kita, M., Kato, M., Ban, Y., Honda, C., Yaegaki, H., Ikoma, Y., \& Moriguchi, T. (2007). Carotenoid accumulation in Japanese apricot (Prunus mume Siebold \& Zucc.): molecular analysis of carotenogenic gene expression and ethylene regulation. J Agric Food Chem, 55, 3414-3420. https://pubs.acs.org/doi/abs/10.1021/jf063552v

Klee, H., \& Tieman, D. (2002). The tomato ethylene receptor gene family: Form and function. Physiol Plant, 115, 336-341. https://doi.org/10.1034/j.1399-3054.2002.1150302.x

Li, J., Tao, X., Li, L., Mao, L., Luo, Z., Khan, Z. U., \& Ying, T. (2016). Comprehensive RNA-Seq Analysis on the Regulation of Tomato Ripening by Exogenous Auxin. PLoS One, 11, e0156453. https://doi.org/10.1371/journal.pone.0156453.

Liu, Y., Ream, A., Joerger, R. D., Liu, J., \& Wang, Y. (2011). Gene expression profiling of a pressure-tolerant 
Listeria monocytogenes Scott A ctsR deletionmutant. J Ind Microbiol Biotechnol, 38(9), 1523-33. https://doi.org/10.1007/s10295-011-0940-9

Mao, X., Cai, T., Olyarchuk, J. G., \& Wei, L. (2005). Automated genome annotation and pathway identification using the KEGG Orthology (KO) as a controlled vocabulary. Bioinformatics, 21(19), 3787-3793. https://doi.org/10.1093/bioinformatics/bti430

Mou, W., Li, D., Bu, J., Jiang, Y., Khan, Z. U., Luo, Z., Mao, L., \& Ying, T. (2016). Comprehensive Analysis of ABA Effects on Ethylene Biosynthesis and Signaling during Tomato Fruit Ripening. PLoS One, 11, e0154072. https://doi.org/10.1371/journal.pone.0154072

Osorio, S., Scossa, F., \& Fernie, A. R. (2013). Molecular regulation of fruit ripening. Front Plant Sci., 4, 198. https://doi.org/10.3389/fpls.2013.00198

Pfaffl, M. W. (2001). A new mathematical model for relative quantification in real-time RT-PCR. Nucleic Acids Res., 29, e45. https://doi.org/10.1093/nar/29.9.e45

Phan, T. D., Bo, W., West, G., Lycett, G. W., \& Tucker, G. A. (2007). Silencing of the major salt-dependent isoform of pectinesterase in tomato alters fruit softening. Plant Physiology 144, 1960-1967. https://doi.org/10.1104/pp.107.096347

Sato, S., Tabata, S., Hirakawa, H., Asamizu, E., Shirasawa, K., Isobe, S., ... Aoki, K. (2012). The tomato genome sequence provides insights into fleshy fruit evolution. Nature, 485, 635-641. http://dx.doi.org/10.1038/nature11119

Serek, M., Tamari, G., Sisler, E., \& Borochov, A. (1995). Inhibition of ethylene-induced cellular senescence symptoms by 1-methylcyclopropene, a new inhibitor of ethylene action. Physiol Plantarum, 94, 229-232. https://doi.org/10.1111/j.1399-3054.1995.tb05305.x

Shen, Y. H., Lu, B. G., Feng, L., Yang, F. Y., Geng, J. J., Ming, R., \& Chen, X. J. (2017). Isolation of ripening-related genes from ethylene/1-MCP treated papaya through RNA-seq. BMC Genomics, 18, 671. https://doi.org/10.1186/s12864-017-4072-0

Sisler, E. C. (2006). The discovery and development of compounds counteracting ethylene at the receptor level. Biotechnol Adv., 24, 357-367. https://doi.org/10.1016/j.biotechadv.2006.01.002

Tang, N., Deng, W., Hu, G., Hu, N., \& Li, Z. (2015). Transcriptome profiling reveals the regulatory mechanism underlying pollination dependent and parthenocarpic fruit set mainly mediated by auxin and gibberellin. PLoS One, 10, e0125355. https://doi.org/10.1371/journal.pone.0125355

Tassoni, A., Watkins, C. B., \& Davies, P. J. (2006). Inhibition of the ethylene response by 1-MCP in tomato suggests that polyamines are not involved in delaying ripening, but may moderate the rate of ripening or over-ripening. J Exp Bot., 57, 3313-3325. https://doi.org/10.1093/jxb/erl092

Trapnell, C., Williams, B. A., Pertea, G., Mortazavi, A., Kwan, G., Van Baren, M. J., Salzberg, S. L., Wold, B. J., \& Pachter, L. (2010). Transcript assembly and quantification by RNA - Seq reveals unannotated transcripts and isoform switching during cell differentiation. Nat Biotechnol, 28, 511-515. http://dx.doi.org/10.1038/nbt.1621

Wang, Z., Gerstein, M., \& Snyder, M. (2009). RNA-Seq: a revolutionary tool for transcriptomics. Nat Rev Genet, 10, 57-63. http://dx.doi.org/10.1038/nrg2484

Ye, J., Hu, T., Yang, C., Li, H., Yang, M., Ijaz, R., Ye, Z., \& Zhang, Y. (2015). Transcriptome Profiling of Tomato Fruit Development Reveals Transcription Factors Associated with Ascorbic Acid, Carotenoid and Flavonoid Biosynthesis. PLoS One, 10, e0130885. https://doi.org/10.1371/journal.pone.0130885

Zhang, J., Cheng, D., Wang, B., Khan, I., \& Ni, Y. (2017). Ethylene Control Technologies in Extending Postharvest Shelf Life of Climacteric Fruit. J Agric Food Chem., 65, 7308-7319. https://doi.org/10.1021/acs.jafc.7b02616

\section{Copyrights}

Copyright for this article is retained by the author(s), with first publication rights granted to the journal.

This is an open-access article distributed under the terms and conditions of the Creative Commons Attribution license (http://creativecommons.org/licenses/by/4.0/). 